\title{
Vandetanib alters the protein pattern in malignant glioma and normal brain in the $\mathrm{BT} 4 \mathrm{C}$ rat glioma model
}

\author{
CARL WIBOM $^{1-3}$, MARIA SANDSTRÖM ${ }^{1}$, ROGER HENRIKSSON ${ }^{1}$, HENRIK ANTTI ${ }^{3}$, \\ MIKAEL JOHANSSON ${ }^{1}$ and A. TOMMY BERGENHEIM ${ }^{2}$ \\ Departments of ${ }^{1}$ Oncology and ${ }^{2}$ Neurosurgery, Umeå University Hospital; \\ ${ }^{3}$ Department of Chemistry, Umeå University, Umeå, Sweden
}

Received April 14, 2010; Accepted June 1, 2010

DOI: 10.3892/ijo_00000739

\begin{abstract}
The treatment of glioblastoma is unsatisfactory. Improved understanding of the biological effects of treatment, together with development of new tools to predict outcome of the initiated treatment are therefore of great need. Vandetanib (ZD6474) is mainly a vascular endothelial growth factor (VEGF) and epidermal growth factor (EGF) receptor tyrosine kinase inhibitor. This study investigated the pattern of protein expression in brain tumor and normal brain tissue, following treatment with vandetanib in a rat glioma model. BT4C-cells were stereotactically implanted into the brain of BD IX rats. The rats were divided into three different experiments. The treatment schedule for experiments one and two consisted of daily, oral doses of vandetanib from day 6 until day 12 or 20 after implantation, respectively. In the third experiment, each animal received a single dose of vandetanib on day 19 after implantation and was then sacrificed 2, 8 or 24 h thereafter. The protein expression profiles were analyzed by SELDITOF-MS and evaluated with multivariate statistical methods. Following treatment with vandetanib, we found significantly altered protein expression pattern in malignant glioma and normal brain. Analyzing protein spectra is an interesting option to assess biological effects induced in brain tissue by signal transduction inhibitors such as vandetanib.
\end{abstract}

\section{Introduction}

Glioblastoma is the most common primary brain tumor and the prognosis for a patient diagnosed with this disease is still

Correspondence to: Dr Carl Wibom, Department of Radiation Sciences, Oncology, Umeå University, S-901 85 Umeå, Sweden E-mail: carl.wibom@onkologi.umu.se

Abbreviations: EGF, endothelial growth factor; VEGF, vascular endothelial growth factor; QC, quality control; EAM, energy absorbing molecule; UV, unit variance; OPLS-DA, orthogonal partial least squares discriminant analysis; CEC, circulating endothelial cells; CEP, circulating endothelial progenitor cells

Key words: angiogenesis, biomarkers, glioblastoma, proteomic pattern, vandetanib poor. However, the outcome has increased significantly by modern multimodal treatment, consisting of surgery, radiochemotherapy and adjuvant chemotherapy, leading to a median survival of 16 months and a 2-year survival of almost $30 \%$ (1). Due to its infiltrative growth, glioblastoma may be considered a generalized central nervous system disease and therefore new systemic treatments are necessary to improve treatment outcome.

Neovascularisation is commonly regarded as one of the hallmarks of cancer (2). Extensive angiogenesis is associated with worse prognosis in glioblastoma $(3,4)$ and vascular endothelial growth factor (VEGF) is reported to be one of the key positive regulators of angiogenesis in brain tumors $(5,6)$. Different strategies for anti-angiogenesis treatment have shown promise in the experimental setting for malignant glioma (7). These include antisense-VEGF strategies (8), anti-VEGF monoclonal antibodies (9), anti-VEGF-receptor (VEGFR) monoclonal antibodies (10), soluble VEGF decoy receptors (11), and small molecule VEGFR tyrosine kinase inhibitors (12-15). Recent phase II clinical data indicate that the anti-VEGF monoclonal antibody bevacizumab is also active in human glioblastoma (16-18).

Vandetanib (ZD6474) is a small molecule inhibitor of the tyrosine kinase of VEGFR2, VEGFR3 and epidermal growth factor receptor (EGFR) (19). Vandetanib has shown promising effects on tumor growth in a panel of subcutaneous tumor models (20) as well as in an intracranial rat glioma model (13). The molecule is now in clinical trials for various tumors, including glioma. This compound has recently shown interesting combination effects when given together with radiotherapy or temozolomide in an orthotopic rat glioma model (21). Due to its dual action as an inhibitor of angiogenesis as well as EGFR driven tumor cell proliferation, it is an interesting compound for glioma treatment.

One clinical problem in the evaluation of new systemic treatments for cancer in general and brain tumors in particular is the need for new ways to predict treatment response. Present treatment regimens have usually been evaluated using conventional radiological methods several months after treatment initiation. The development of new biological and radiological methods for detection of early treatment response is of great importance when an increasing number of new signal transduction inhibitors are to be evaluated. One step in this direction is to investigate the effect of a given treatment 
based on the protein expression pattern. With respect to glioma treatment this has previously been performed in cell culture systems $(22,23)$. In the present study, the pattern of protein expression in an experimental in vivo glioma model following treatment with the angiogenesis inhibitor vandetanib was evaluated, using surface enhanced laser desorption/ ionization time of flight mass spectrometry (SELDI-TOF-MS).

\section{Materials and methods}

Animal model. The BT4C syngenic intracerebral rat glioma model was used for the in vivo experiments in this study $(24,25)$. The BT4C rat glioma cells were grown in Dulbecco's modification of Eagle's MEM (DMEM) (Gibco/Invitrogen Corp., Paisley, Scotland, UK) supplied with 5\% BD-IX fetal calf serum. When grown in log-phase the cells were harvested and diluted to a concentration of 4,000 cells $/ \mu 1$. A $22 \mathrm{G}$ microsyringe (Unimetrics, Shorewood, IL) fitted to a stereotactic frame was used to inject $5 \mu 1$ of the cell suspension into the right caudate nucleus of the inbred BD IX rats. Care was taken to prevent reflux in the insertion canal and burr hole by a slow injection and the use of bone wax. During the implantation procedure, cells were kept on ice and the viability was monitored by intermittent tryphan-blue staining. The rats were anaesthetized with a 1:1 mixture of $\mathrm{Hypnorm}^{\circledR}$ (fluanisone $10 \mathrm{mg} / \mathrm{ml}$ and fentanyl citrate $0.315 \mathrm{mg} / \mathrm{ml}$ ) and Dormicum ${ }^{\circledR}$ (midazolam $5 \mathrm{mg} / \mathrm{ml}$ ) in the dose $0.5 \mathrm{ml} / 100 \mathrm{~g}$. In addition, local anaesthesia (Xylocain ${ }^{\circledR} 10 \mathrm{mg} / \mathrm{ml}$ ) was used in the scalp.

The animals were provided with food and water ad libitum and were housed in a controlled environment with 12-h light/dark cycles. An experienced animal keeper supervised the care of the animals.

Treatment. In total, 27 animals were used. The animals were divided into three experiments with different times of initiation and duration of treatment (Fig. 1). The first two experiments each consisted of four rats receiving treatment, daily oral dosages of $50 \mathrm{mg} / \mathrm{kg}$ vandetanib, and three untreated control animals, respectively. Treatment for both these experiments commenced on day 6 after implantation and lasted for 6 and 14 days in experiment 1 and 2 respectively. Hence all animals in experiment 1 were sacrificed on day 12 after implantation and all animals in experiment 2 on day 20 after implantation. All 9 animals in the third experiment were treated with a single dose of $50 \mathrm{mg} / \mathrm{kg}$ vandetanib on day 19 after implantation and then sacrificed in groups of three; 2, 8 , and $24 \mathrm{~h}$ after treatment. To save animals, the untreated animals from experiment 3 served as a control group also for this experiment. In addition, 4 tumor-free, sham-operated animals were included in the study. These animals were subject to the same surgical procedure as all the other animals, except that saline was injected instead of tumor cells.

After decapitation, tumors were grossly dissected together with contralateral normal brain tissue. Tissue was snap-frozen in liquid nitrogen and stored at $-80^{\circ} \mathrm{C}$. The experiment was approved by the Umeå University ethics committee for animal research.

Vandetanib (ZD6474). Vandetanib was kindly provided by AstraZeneca, Alderley Park, UK. For details and chemical structure see Hennequin et al (19). Vandetanib was dissolved according to the manufacturer's instructions in $1 \%$ Tween-80 (Merck-Schuchardt, Hohenbrunn, Germany) to a concentration of $10 \mathrm{mg} / \mathrm{ml}$. Glass beads were added to the mixture to obtain a uniform suspension. The suspension was then milled overnight at room temperature.

Tissue preparation. To allow for protein profiling analyses, each tissue sample was homogenized in a series of steps. The sequence in which the samples were handled was randomized to avoid systematic differences as a result of sample treatment. Each sample was firstly thoroughly homogenized in $1 \mathrm{ml}$ homogenization buffer 1 (100 mM HEPES, pH 7.4, $100 \mathrm{mM}$ $\mathrm{NaCl}, 0.5 \%$ CHAPS) per $100 \mathrm{mg}$ tissue, with a Dounce Tissue Grinder (Kimble/Kontes, Vineland, NJ, USA), and then incubated on ice for $30 \mathrm{~min}$. Thereafter, the homogenate was centrifuged at $20,800 \mathrm{~g}$ for $20 \mathrm{~min}$ at $4^{\circ} \mathrm{C}$ and both the supernatant and the pellet fraction were recovered. The supernatant was dissolved in two volumes of protein denaturing buffer (8 M urea, 1\% CHAPS, PBS), kept on a shaker for $30 \mathrm{~min}$ at $4^{\circ} \mathrm{C}$ and then snap-frozen in liquid nitrogen and stored at $-80^{\circ} \mathrm{C}$. The pellet fraction was transferred back to the tissue grinder and rehomogenized in $1 \mathrm{ml}$ homogenization buffer 2 [5 M Guanidine-HCl, $50 \mathrm{mM}$ Tris ( $\mathrm{pH} 8.0$ ), $0.5 \%$ CHAPS] per $100 \mathrm{mg}$ tissue, then incubated on ice for $3 \mathrm{~h}$, followed by centrifugation at $20,800 \mathrm{~g}$ for $20 \mathrm{~min}$ at $4^{\circ} \mathrm{C}$ before it was snap-frozen in liquid nitrogen and stored at $-80^{\circ} \mathrm{C}$. Both homogenization buffers contained Complete EDTAfree Protease Inhibitor Cocktail (Roche Applied Science, Indianapolis, IN, USA). The protein concentration of each sample was measured using the bicinchoninic assay (BCA) (Pierce Biotechnology, Inc., Rockford, IL, USA).

Sample analysis. The protein content of each sample was profiled using SELDI-TOF-MS, and certain ProteinChip arrays (Bio-Rad Laboratories Inc., Hercules, CA, USA). The array types used were CM10 (a weak cat ion exchange surface) and IMAC (an immobilized metal affinity chromatography surface) coupled with $\mathrm{Cu}^{2+}$ ions. The analyses were done for one fraction at a time, on one array type at the time, and the samples were applied to the protein chip arrays in triplicates according to a randomized scheme. To facilitate evaluation of analytical reproducibility a number of quality control (QC) samples were analyzed together with the other samples, randomly located to different spots on the ProteinChip arrays. The array preparation strategy has been described in detail elsewhere (26), below follows a short description.

The IMAC arrays were charged two times with $\mathrm{Cu}^{2+}$ ions by addition of $50 \mu \mathrm{l}$ of $100 \mathrm{mM} \mathrm{CuSO}_{4}$ for $5 \mathrm{~min}$. Unbound metal ions were removed by two washes with $100 \mu 11 \mathrm{mM}$ HEPES for $5 \mathrm{~min}$. All subsequent array preparation steps were then identical for both array types. The first of which was equilibration with $150 \mu \mathrm{l}$ array specific binding buffer, where the binding buffers used were $0.1 \mathrm{M} \mathrm{PO}_{4}, \mathrm{pH} 7.5$ with and without $0.5 \mathrm{M} \mathrm{NaCl}$ for the IMAC and the CM10 arrays, respectively. The equilibration step was repeated two more times. In parallel to the pre-treatment of the ProteinChip arrays, the samples were thawed on ice and diluted in binding buffer to a concentration of $150 \mathrm{ng} / \mu 1$. One hundred $\mu 1$ of the diluted samples were added to the pre-treated ProteinChip 


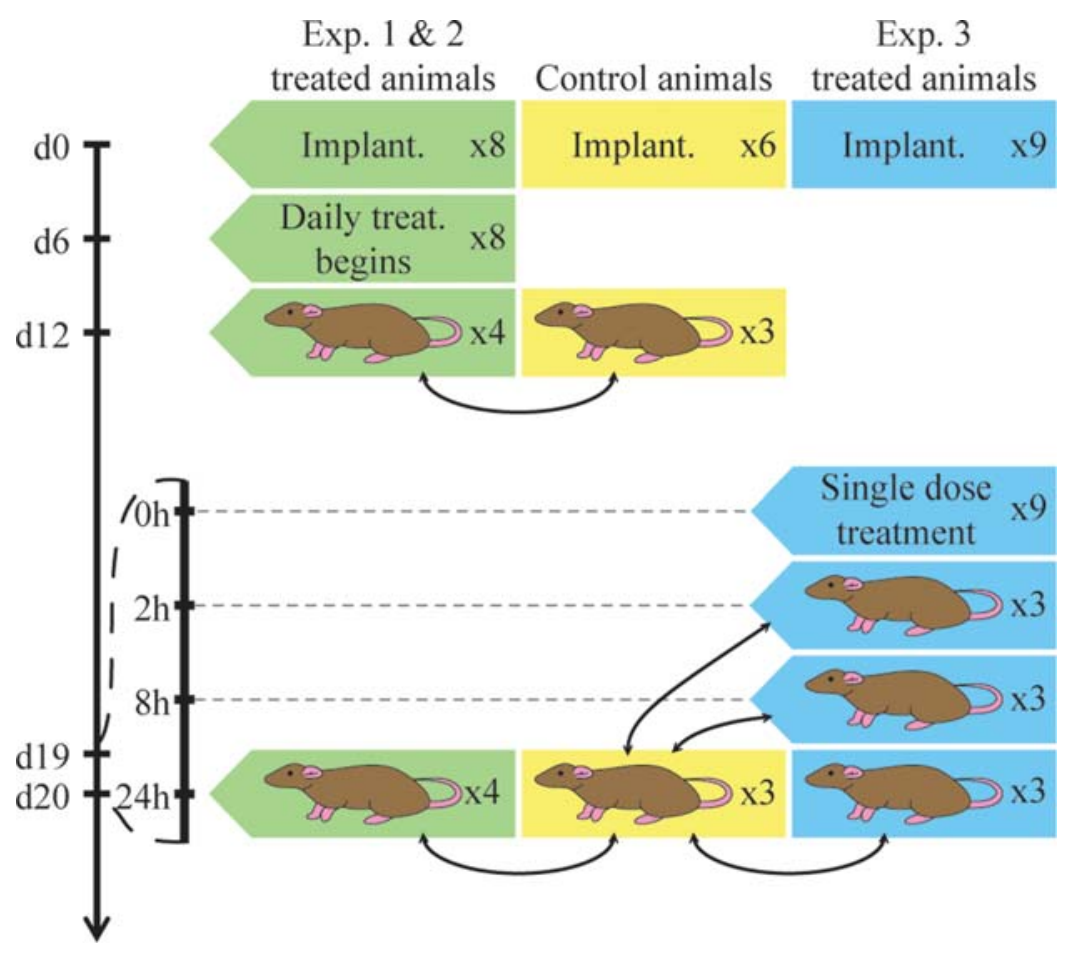

Figure 1. Schematic overview of the study design. The time scale represents days after implantation (d0), and is zoomed in on the time between days 19 and 20 after implantation. The numbers to the right in each box denote the number of animals subjected to a procedure. The rat drawings represent sampling time-points and the arrows connect groups that were compared against each other.

arrays and allowed to incubate for one hour. All arrays were then washed three times with binding buffer for $5 \mathrm{~min}$ and twice with $1 \mathrm{mM}$ HEPES for $1 \mathrm{~min}$. Lastly, the arrays were air-dried for 15 min before addition of the energy absorbing molecule (EAM) matrix. The EAM used was 50\% saturated sinapinic acid, diluted in $50 \%$ acetonitrile and $0.5 \%$ triflouroacetic acid, which was added in two deliveries of $1 \mu \mathrm{l}$ each, 3 min apart.

The array preparation was carried out in room temperature using the Biomek 2000 Laboratory Automation Workstation robot (Beckman Coulter Inc., Fullerton, CA, USA), coupled with a MicroMix 5 shaker (Diagnostic Products Corp., Los Angeles, CA, USA), which was used for all incubations, set to program 5 and amplitude 20 . The arrays were subsequently analyzed twice in the TOF mass spectrometer (PBS-II; Bio-Rad Laboratories Inc.), with different settings optimized for different mass regions; 1.8-10 and 10-20 kDa. In addition, the QC-samples were also analyzed with settings optimized for a wider mass region: $1.8-20 \mathrm{kDa}$.

Analytical reproducibility. Spectra from the QC samples were used to evaluate the analytical reproducibility, and therefore treated separately from the rest. The QC spectra were baseline subtracted and then normalized together in the ProteinChip Software v3.2.2 (Ciphergen Biosystems Inc., Fremont, CA, USA). Clusters of peaks were selected within the mass region 2.5-20 kDa, using the Biomarker Wizard tool with the following settings; first pass signal to noise ratio $=$ 5 , min peak threshold $=50 \%$ of all spectra, cluster mass window $=0.3 \%$ of mass, second pass signal to noise ratio $=$ 2 , with the noise set to be calculated within the same mass region. A coefficient of variance $(\mathrm{CV})$ value was calculated for each peak cluster individually, and the mean of each peak cluster's CV was used as a measure of the reproducibility.

Data pre-processing. Following baseline subtraction, which was carried out using the ProteinChip Software, spectra from both analyzed mass regions for each sample were exported. To allow for meaningful comparison between spectra from different samples, the exported data were subject to a binning procedure, using 2.5 and $3.5 \mathrm{Da}$ as bin sizes for the 1.8-10 and the 10-20 kDa regions, respectively. Each spectrum was then normalized by total ion current (TIC), i.e., each bin variable was divided by the mean of all bin variables in the mass region. Prior to multivariate data analysis the data were mean centered, and scaled by either pareto- or unit variance (UV) scaling.

Data analyses. The use of two separate sample fractions, each analyzed on two different ProteinChip array types at two different mass regions, gave rise to 8 different data subsets in total. The complete protein profiles from each subset were pre-processed and assembled into separate data tables denoted as $\mathbf{X}$-matrices.

Each X-matrix was initially scanned for possible outliers, i.e., deviating samples not suitable to be included in further analysis. This was done by means of principal component analysis (PCA). Samples detected as outliers, including spectra of inadequately low quality and possibly contaminated samples, were excluded. Following PCA, the $\mathbf{X}$-matrix was pareto scaled and analyzed by orthogonal partial least squares discriminant analysis (OPLS-DA) (27) versus a response matrix $(\mathbf{Y})$, containing three dummy variables describing tissue type (normal or tumor), treatment and the interaction 
Table I. Peaks significantly altered by treatment, found in the pellet fraction analysed on CM10 arrays.

\begin{tabular}{|c|c|c|c|c|c|c|}
\hline \multirow[b]{2}{*}{$\mathrm{m} / \mathrm{z}^{\mathrm{a}}$} & \multicolumn{6}{|c|}{ CM10/pellet } \\
\hline & Time $^{\mathrm{b}}$ & Tissue $^{c}$ & int_ctrl ${ }^{\mathrm{d}}$ & int_zde & p-value ${ }^{f}$ & Permutation ${ }^{\mathrm{g}}$ \\
\hline 2576.2 & $24 \mathrm{~h}$ & $\mathrm{~T}$ & 2.34 & 0.11 & 0.001 & - \\
\hline 3132.5 & $24 \mathrm{~h}$ & $\mathrm{~T}$ & 2.68 & 0.42 & 0.001 & - \\
\hline 3410.7 & $6 \mathrm{~d}$ & $\mathrm{~T}$ & 1.74 & 0.62 & 0.000 & $\mathrm{x}$ \\
\hline 3467.9 & $14 \mathrm{~d}$ & $\mathrm{~N}$ & 1.08 & 0.31 & 0.000 & $\mathrm{x}$ \\
\hline \multirow[t]{2}{*}{3871.7} & $14 \mathrm{~d}$ & $\mathrm{~N}$ & 1.72 & 0.53 & 0.004 & - \\
\hline & $14 \mathrm{~d}$ & $\mathrm{~T}$ & 0.89 & 1.98 & 0.001 & - \\
\hline 3955.1 & $6 \mathrm{~d}$ & $\mathrm{~T}$ & 3.33 & 1.91 & 0.000 & $\mathrm{x}$ \\
\hline \multirow[t]{2}{*}{4341.1} & $2 \mathrm{~h}$ & $\mathrm{~T}$ & 2.47 & 5.57 & 0.001 & - \\
\hline & $8 \mathrm{~h}$ & $\mathrm{~T}$ & 2.47 & 5.38 & 0.008 & - \\
\hline 4468.9 & $8 \mathrm{~h}$ & $\mathrm{~T}$ & 4.92 & 9.83 & 0.000 & - \\
\hline 5005.5 & $2 \mathrm{~h}$ & $\mathrm{~T}$ & 1.03 & 3.84 & 0.008 & - \\
\hline \multirow[t]{2}{*}{5178.2} & $2 \mathrm{~h}$ & $\mathrm{~T}$ & 1.17 & 0.45 & 0.006 & - \\
\hline & $8 \mathrm{~h}$ & $\mathrm{~T}$ & 1.17 & 0.36 & 0.002 & - \\
\hline \multirow[t]{2}{*}{5580.9} & $8 \mathrm{~h}$ & $\mathrm{~N}$ & 2.19 & 1.22 & 0.006 & - \\
\hline & $14 \mathrm{~d}$ & $\mathrm{~N}$ & 2.19 & 0.87 & 0.000 & $\mathrm{x}$ \\
\hline 6614.0 & $14 \mathrm{~d}$ & $\mathrm{~T}$ & 1.70 & 3.91 & 0.000 & $\mathrm{x}$ \\
\hline \multirow[t]{3}{*}{6720.9} & $14 \mathrm{~d}$ & $\mathrm{~N}$ & 6.10 & 3.16 & 0.002 & - \\
\hline & $14 \mathrm{~d}$ & $\mathrm{~T}$ & 2.37 & 4.42 & 0.000 & - \\
\hline & $8 \mathrm{~h}$ & $\mathrm{~N}$ & 6.10 & 3.30 & 0.003 & - \\
\hline \multirow[t]{2}{*}{7623.2} & $24 \mathrm{~h}$ & $\mathrm{~N}$ & 2.96 & 4.52 & 0.006 & - \\
\hline & $24 \mathrm{~h}$ & $\mathrm{~T}$ & 0.93 & 2.98 & 0.008 & - \\
\hline 8685.0 & $2 \mathrm{~h}$ & $\mathrm{~T}$ & 0.25 & 1.33 & 0.002 & - \\
\hline 9836.2 & $2 \mathrm{~h}$ & $\mathrm{~T}$ & 2.97 & 9.73 & 0.008 & - \\
\hline 10660.5 & $8 \mathrm{~h}$ & $\mathrm{~T}$ & 0.67 & 1.18 & 0.006 & - \\
\hline
\end{tabular}

${ }^{a}$ Mean $\mathrm{m} / \mathrm{z}$ value of each peak. ${ }^{\mathrm{b}}$ Duration of treatment. ${ }^{\mathrm{c}} \mathrm{N}$ and $\mathrm{T}$ denote normal and tumor, respectively. ${ }^{\mathrm{d}}$ Mean normalized intensity for untreated samples. ${ }^{e}$ Mean normalized intensity for treated samples. ${ }^{\mathrm{f}} \mathrm{p}$-values were calculated by Mann-Whitney $\mathrm{U}$ statistics for each peak. ${ }^{g} \mathrm{X}$ denotes that it was found significant by permutation and - denotes that it was not.

term between these two variables. This analysis resulted in three predictive OPLS-components. The correlation loading vectors $[\mathbf{p}(\mathbf{c o r r})]$ and the covariance loading vectors $\left(\mathbf{w}^{*}\right)$ from each component were subsequently used to define spectral regions of interest (ROI), i.e. regions where consecutive bin-variables displayed either large absolute $\mathbf{p}($ corr $)$-values $(\mid \mathbf{p}($ corr $) \mid>0.5)$ or large absolute $\mathbf{w}^{*}$-values $\left(\left|\mathbf{w}^{*}\right|>0.025\right)$. Variables within ROI that constituted local maxima (peaks) in the mean protein profile from any of the compared groups were then located, and their intensity data extracted from all individual observations. This was done by identifying local maximum intensities in each observation's profile within $\pm 0.2 \%$ of the $\mathrm{m} / \mathrm{z}$-value defined as a peak in the mean spectrum in order to compensate for possible mass shifts. Peaks with a normalized mean intensity $<1$ in both groups were discarded, together with peaks displaying a resolution $<100$ in both groups, as calculated by Full Width at Half Maximum on the mean profile.

The procedure described above was repeated for all eight data subsets, and the extracted data from all subsets were compiled in a new $\mathbf{X}$-matrix $\left(\mathbf{X}_{\mathbf{b}}\right)$. The variables in $\mathbf{X}_{\mathbf{b}}$ were
UV-scaled prior to OPLS-DA analysis against the same response matrix $(\mathbf{Y})$ as described above. The $\mathbf{p}(\mathbf{c o r r})$-vectors from the components describing treatment effects in normal and tumor samples, respectively, were used to locate the peaks most notably affected by treatment in any of the tissue types. Peaks with an absolute p(corr)-value $>0.5$ in at least one of the vectors were considered as interesting.

In addition, a permutation analysis was employed to locate peaks affected by treatment. This was done for one tissue type at a time, where the correlation between each peaks' expression pattern $(\mathbf{X b})$ and a response variable $(\mathbf{y})$, representing treatment, was calculated. The response variable was then randomly permutated, and a new correlation value was calculated. This was repeated 10,000 times, and variables with a greater correlation to the original $\mathbf{y}$ than any of the permuted $\mathbf{y}$-variables were considered as interesting. The permutation procedure was repeated three times and the variables surviving the test at least once were included for further evaluation.

Finally, all selected peaks were confirmed by manual inspection of the spectra and validated using a Mann-Whitney 
Table II. Peaks significantly altered by treatment, found in the supernatant fraction analysed on CM10 arrays.

\begin{tabular}{|c|c|c|c|c|c|c|}
\hline \multirow[b]{2}{*}{$\mathrm{m} / \mathrm{z}^{\mathrm{a}}$} & \multicolumn{6}{|c|}{ CM10/supernatant } \\
\hline & Time $^{\mathrm{b}}$ & Tissue $^{c}$ & int_ctrl ${ }^{\mathrm{d}}$ & int_zd ${ }^{e}$ & p-value ${ }^{f}$ & Permutation ${ }^{\mathrm{g}}$ \\
\hline 2678.8 & $6 \mathrm{~d}$ & $\mathrm{~T}$ & 5.22 & 3.48 & 0.000 & $\mathrm{x}$ \\
\hline 2894.9 & $14 \mathrm{~d}$ & $\mathrm{~N}$ & 1.29 & 0.60 & 0.000 & $\mathrm{x}$ \\
\hline 3061.4 & $14 \mathrm{~d}$ & $\mathrm{~T}$ & 1.21 & 0.30 & 0.000 & $\mathrm{x}$ \\
\hline \multirow[t]{2}{*}{3262.5} & $24 \mathrm{~h}$ & $\mathrm{~N}$ & 0.63 & 1.09 & 0.000 & $\mathrm{x}$ \\
\hline & $14 \mathrm{~d}$ & $\mathrm{~N}$ & 0.63 & 1.66 & 0.000 & $\mathrm{x}$ \\
\hline 3310.5 & $14 \mathrm{~d}$ & $\mathrm{~N}$ & 1.52 & 4.07 & 0.000 & $\mathrm{x}$ \\
\hline 3497.7 & $14 \mathrm{~d}$ & $\mathrm{~T}$ & 3.33 & 1.24 & 0.000 & $\mathrm{x}$ \\
\hline 3871.7 & $14 \mathrm{~d}$ & $\mathrm{~T}$ & 2.25 & 5.40 & 0.009 & - \\
\hline 4106.3 & $2 \mathrm{~h}$ & $\mathrm{~N}$ & 1.59 & 1.07 & 0.000 & $\mathrm{x}$ \\
\hline 4163.5 & $6 \mathrm{~d}$ & $\mathrm{~T}$ & 1.86 & 1.11 & 0.008 & - \\
\hline 4235.8 & $6 \mathrm{~d}$ & $\mathrm{~N}$ & 5.40 & 3.91 & 0.007 & - \\
\hline \multirow[t]{2}{*}{4683.2} & $14 \mathrm{~d}$ & $\mathrm{~N}$ & 3.17 & 1.32 & 0.000 & $\mathrm{x}$ \\
\hline & $2 \mathrm{~h}$ & $\mathrm{~N}$ & 3.17 & 1.80 & 0.008 & - \\
\hline 5098.1 & $6 \mathrm{~d}$ & $\mathrm{~T}$ & 34.61 & 21.88 & 0.000 & $\mathrm{x}$ \\
\hline 5240.9 & $8 \mathrm{~h}$ & $\mathrm{~N}$ & 2.19 & 3.88 & 0.006 & - \\
\hline 5316.3 & $14 \mathrm{~d}$ & $\mathrm{~T}$ & 2.05 & 1.31 & 0.002 & - \\
\hline 5436.6 & $14 \mathrm{~d}$ & $\mathrm{~T}$ & 3.69 & 1.07 & 0.000 & $\mathrm{x}$ \\
\hline \multirow[t]{2}{*}{5521.3} & $14 \mathrm{~d}$ & $\mathrm{~N}$ & 5.36 & 3.04 & 0.000 & $\mathrm{x}$ \\
\hline & $14 \mathrm{~d}$ & $\mathrm{~T}$ & 1.46 & 0.83 & 0.006 & - \\
\hline \multirow[t]{4}{*}{5649.1} & $14 \mathrm{~d}$ & $\mathrm{~N}$ & 0.97 & 4.31 & 0.000 & $\mathrm{x}$ \\
\hline & $14 \mathrm{~d}$ & $\mathrm{~T}$ & 2.64 & 1.47 & 0.002 & - \\
\hline & $24 \mathrm{~h}$ & $\mathrm{~N}$ & 0.97 & 1.91 & 0.001 & - \\
\hline & $24 \mathrm{~h}$ & $\mathrm{~T}$ & 2.48 & 4.67 & 0.003 & - \\
\hline 5653.1 & $2 \mathrm{~h}$ & $\mathrm{~N}$ & 1.00 & 2.01 & 0.000 & - \\
\hline \multirow[t]{3}{*}{5714.7} & $24 \mathrm{~h}$ & $\mathrm{~T}$ & 6.28 & 3.13 & 0.008 & - \\
\hline & $14 \mathrm{~d}$ & $\mathrm{~N}$ & 6.45 & 4.76 & 0.009 & - \\
\hline & $14 \mathrm{~d}$ & $\mathrm{~T}$ & 6.88 & 2.64 & 0.000 & $\mathrm{x}$ \\
\hline 6150.6 & $24 \mathrm{~h}$ & $\mathrm{~T}$ & 0.83 & 1.63 & 0.005 & - \\
\hline \multirow[t]{3}{*}{6287.6} & $14 \mathrm{~d}$ & $\mathrm{~N}$ & 3.90 & 8.42 & 0.000 & $\mathrm{x}$ \\
\hline & $24 \mathrm{~h}$ & $\mathrm{~N}$ & 3.92 & 5.63 & 0.001 & - \\
\hline & $24 \mathrm{~h}$ & $\mathrm{~T}$ & 0.52 & 4.40 & 0.001 & - \\
\hline 7915.6 & $24 \mathrm{~h}$ & $\mathrm{~N}$ & 0.89 & 1.29 & 0.001 & - \\
\hline 9815.7 & $6 \mathrm{~d}$ & $\mathrm{~T}$ & 1.11 & 0.40 & 0.008 & - \\
\hline 10094.1 & $14 \mathrm{~d}$ & $\mathrm{~T}$ & 5.09 & 8.99 & 0.009 & - \\
\hline 10292.3 & $24 \mathrm{~h}$ & $\mathrm{~T}$ & 4.78 & 7.03 & 0.005 & - \\
\hline \multirow[t]{2}{*}{10660.0} & $8 \mathrm{~h}$ & $\mathrm{~N}$ & 1.75 & 3.05 & 0.000 & - \\
\hline & $14 \mathrm{~d}$ & $\mathrm{~N}$ & 1.81 & 4.44 & 0.000 & $\mathrm{x}$ \\
\hline 12403.8 & $8 \mathrm{~h}$ & $\mathrm{~N}$ & 3.98 & 1.88 & 0.003 & - \\
\hline
\end{tabular}

${ }^{\mathrm{a}}$ Mean $\mathrm{m} / \mathrm{z}$ value of each peak. ${ }^{\mathrm{b}}$ Duration of treatment. ${ }^{\mathrm{c}} \mathrm{N}$ and $\mathrm{T}$ denote normal and tumor, respectively. ${ }^{\mathrm{d}}$ Mean normalized intensity for untreated samples. ${ }^{\mathrm{e}}$ Mean normalized intensity for treated samples. ${ }^{\mathrm{f}} \mathrm{p}$-values were calculated by Mann-Whitney U statistics for each peak. ${ }^{\mathrm{g}} \mathrm{X}$ denotes that it was found significant by permutation and - denotes that it was not.

U test to generate a p-value. Two final, UV-scaled OPLS-DA models were then calculated, using a single response variable (y) representing treatment; one for samples from normal tissue based on peaks displaying significant $(\mathrm{p}<0.01)$ treatment effects in normal tissue, and one for tumor samples based on peaks significantly affected in tumor tissue. To visualize the altered expression pattern in normal and tumor tissue respectively in a reliable fashion, cross-validated (CV) scores plots were used.

Data from each of the three experiments were analyzed separately, and for each significantly affected peak an exact mass value was extracted from the raw data. The results were complied into 4 separate tables (Tables I-IV), one for each 
Table III. Peaks significantly altered by treatment, found in the pellet fraction analysed on IMAC arrays.

\begin{tabular}{|c|c|c|c|c|c|c|}
\hline \multirow[b]{2}{*}{$\mathrm{m} / \mathrm{z}^{\mathrm{a}}$} & \multicolumn{6}{|c|}{ IMAC/pellet } \\
\hline & Time $^{\mathrm{b}}$ & Tissue $^{c}$ & int_ctrl ${ }^{\mathrm{d}}$ & int_zd ${ }^{\mathrm{e}}$ & $p$-value ${ }^{f}$ & Permutation $^{g}$ \\
\hline \multirow[t]{2}{*}{1996.5} & $14 \mathrm{~d}$ & $\mathrm{~N}$ & 2.08 & 3.26 & 0.001 & - \\
\hline & $14 \mathrm{~d}$ & $\mathrm{~T}$ & 0.67 & 1.13 & 0.002 & $\mathrm{x}$ \\
\hline \multirow[t]{2}{*}{2063.2} & $14 \mathrm{~d}$ & $\mathrm{~N}$ & 1.60 & 2.61 & 0.000 & - \\
\hline & $14 \mathrm{~d}$ & $\mathrm{~T}$ & 0.35 & 0.75 & 0.002 & $\mathrm{x}$ \\
\hline 2200.6 & $14 \mathrm{~d}$ & $\mathrm{~N}$ & 0.57 & 1.25 & 0.000 & $\mathrm{x}$ \\
\hline 2226.2 & $6 \mathrm{~d}$ & $\mathrm{~N}$ & 2.97 & 2.37 & 0.007 & - \\
\hline \multirow[t]{2}{*}{2253.7} & $6 \mathrm{~d}$ & $\mathrm{~N}$ & 3.23 & 2.19 & 0.002 & - \\
\hline & $2 \mathrm{~h}$ & $\mathrm{~N}$ & 1.53 & 2.20 & 0.001 & - \\
\hline \multirow[t]{3}{*}{2308.6} & $14 \mathrm{~d}$ & $\mathrm{~N}$ & 2.55 & 4.13 & 0.004 & - \\
\hline & $14 \mathrm{~d}$ & $\mathrm{~T}$ & 0.92 & 1.41 & 0.002 & $\mathrm{x}$ \\
\hline & $6 \mathrm{~d}$ & $\mathrm{~N}$ & 4.06 & 2.89 & 0.001 & - \\
\hline 2358.1 & $8 \mathrm{~h}$ & $\mathrm{~T}$ & 1.97 & 1.15 & 0.000 & $\mathrm{x}$ \\
\hline \multirow[t]{2}{*}{2404.8} & $14 \mathrm{~d}$ & $\mathrm{~N}$ & 2.08 & 3.45 & 0.002 & - \\
\hline & $14 \mathrm{~d}$ & $\mathrm{~T}$ & 0.67 & 1.25 & 0.002 & $\mathrm{x}$ \\
\hline \multirow[t]{2}{*}{2771.3} & $14 \mathrm{~d}$ & $\mathrm{~N}$ & 1.66 & 3.02 & 0.000 & $\mathrm{x}$ \\
\hline & $8 \mathrm{~h}$ & $\mathrm{~N}$ & 1.66 & 2.69 & 0.006 & - \\
\hline 2843.4 & $2 \mathrm{~h}$ & $\mathrm{~T}$ & 3.22 & 2.01 & 0.008 & - \\
\hline 3043.9 & $8 \mathrm{~h}$ & $\mathrm{~T}$ & 5.02 & 1.40 & 0.000 & $\mathrm{x}$ \\
\hline \multirow[t]{3}{*}{3117.9} & $8 \mathrm{~h}$ & $\mathrm{~T}$ & 1.99 & 1.08 & 0.003 & - \\
\hline & $14 \mathrm{~d}$ & $\mathrm{~N}$ & 0.84 & 1.37 & 0.001 & - \\
\hline & $14 \mathrm{~d}$ & $\mathrm{~T}$ & 1.99 & 0.83 & 0.004 & - \\
\hline 3218.8 & $14 \mathrm{~d}$ & $\mathrm{~N}$ & 0.63 & 1.25 & 0.000 & - \\
\hline 3412.1 & $6 \mathrm{~d}$ & $\mathrm{~T}$ & 1.23 & 0.54 & 0.001 & $\mathrm{x}$ \\
\hline 3476.7 & $6 \mathrm{~d}$ & $\mathrm{~T}$ & 1.59 & 0.77 & 0.000 & $\mathrm{x}$ \\
\hline 3507.3 & $8 \mathrm{~h}$ & $\mathrm{~T}$ & 5.43 & 3.71 & 0.000 & - \\
\hline 3669.3 & $14 \mathrm{~d}$ & $\mathrm{~N}$ & 1.13 & 1.79 & 0.001 & - \\
\hline \multirow[t]{2}{*}{4966.5} & $2 \mathrm{~h}$ & $\mathrm{~N}$ & 1.23 & 0.69 & 0.000 & $\mathrm{x}$ \\
\hline & $2 \mathrm{~h}$ & $\mathrm{~T}$ & 1.46 & 0.96 & 0.005 & - \\
\hline \multirow[t]{3}{*}{5493.2} & $14 \mathrm{~d}$ & $\mathrm{~N}$ & 2.06 & 3.77 & 0.000 & $\mathrm{x}$ \\
\hline & $2 \mathrm{~h}$ & $\mathrm{~N}$ & 2.06 & 2.94 & 0.000 & $\mathrm{x}$ \\
\hline & $8 \mathrm{~h}$ & $\mathrm{~N}$ & 2.07 & 2.89 & 0.000 & $\mathrm{x}$ \\
\hline \multirow[t]{2}{*}{5645.1} & $2 \mathrm{~h}$ & $\mathrm{~N}$ & 1.59 & 2.32 & 0.002 & - \\
\hline & $14 \mathrm{~d}$ & $\mathrm{~N}$ & 1.59 & 3.32 & 0.000 & $\mathrm{x}$ \\
\hline 7901.0 & $14 \mathrm{~d}$ & $\mathrm{~N}$ & 0.71 & 1.31 & 0.000 & - \\
\hline
\end{tabular}

${ }^{a}$ Mean $\mathrm{m} / \mathrm{z}$ value of each peak. ${ }^{\mathrm{b}}$ Duration of treatment. ${ }^{\mathrm{c}} \mathrm{N}$ and $\mathrm{T}$ denote normal and tumor, respectively. ${ }^{\mathrm{d}}$ Mean normalized intensity for untreated samples. ${ }^{e}$ Mean normalized intensity for treated samples. ${ }^{\mathrm{f}} \mathrm{p}$-values were calculated by Mann-Whitney U statistics for each peak. $\mathrm{g}_{\mathrm{X}}$ denotes that it was found significant by permutation and - denotes that it was not.

combination of sample fraction and array type. Peaks who's $\mathrm{m} / \mathrm{z}$-values were not significantly different $(\mathrm{p}<0.01)$ were considered to have the same $\mathrm{m} / \mathrm{z}$, and thus listed together on the same row in the result tables. For all multivariate data modeling steps described above, the cross-validation scheme was designed to keep all replicates of one sample excluded in the same CV-round. This has the effect that the presented cross-validated scores plot will present a predictive pattern, where each predicted subject can be considered as unknown to the model.

\section{Results}

Analytical reproducibility. The tissue samples in this study were initially fractionated into two fractions, they were both subsequently analyzed on two separate types of ProteinChip 
Table IV. Peaks significantly altered by treatment, found in the supernatant fraction analysed on IMAC arrays.

\begin{tabular}{|c|c|c|c|c|c|c|}
\hline \multirow[b]{2}{*}{$\mathrm{m} / \mathrm{z}^{\mathrm{a}}$} & \multicolumn{6}{|c|}{ IMAC/supernatant } \\
\hline & Time $^{\mathrm{b}}$ & Tissue $^{\mathrm{c}}$ & int_ctrl ${ }^{\mathrm{d}}$ & int_zd ${ }^{e}$ & p-value ${ }^{f}$ & Permutation $^{\mathrm{g}}$ \\
\hline 2454.0 & $14 \mathrm{~d}$ & $\mathrm{~N}$ & 2.21 & 1.41 & 0.009 & - \\
\hline 2644.0 & $14 \mathrm{~d}$ & $\mathrm{~N}$ & 1.41 & 0.63 & 0.000 & $\mathrm{x}$ \\
\hline 2647.6 & $14 \mathrm{~d}$ & $\mathrm{~N}$ & 1.38 & 0.62 & 0.001 & - \\
\hline 2775.6 & $14 \mathrm{~d}$ & $\mathrm{~N}$ & 3.93 & 2.80 & 0.004 & - \\
\hline 2807.2 & $14 \mathrm{~d}$ & $\mathrm{~N}$ & 2.57 & 1.71 & 0.003 & - \\
\hline 3066.7 & $6 \mathrm{~d}$ & $\mathrm{~N}$ & 1.69 & 2.43 & 0.002 & - \\
\hline 3209.6 & $14 \mathrm{~d}$ & $\mathrm{~N}$ & 0.94 & 4.77 & 0.000 & $\mathrm{x}$ \\
\hline 3308.8 & $8 \mathrm{~h}$ & $\mathrm{~N}$ & 3.62 & 6.34 & 0.000 & $\mathrm{x}$ \\
\hline 3508.4 & $8 \mathrm{~h}$ & $\mathrm{~N}$ & 0.77 & 1.17 & 0.000 & $\mathrm{x}$ \\
\hline 4673.1 & $2 \mathrm{~h}$ & $\mathrm{~N}$ & 2.65 & 1.46 & 0.008 & - \\
\hline 5639.8 & $14 \mathrm{~d}$ & $\mathrm{~N}$ & 0.57 & 2.52 & 0.000 & $\mathrm{x}$ \\
\hline 5777.6 & $2 \mathrm{~h}$ & $\mathrm{~T}$ & 1.21 & 3.24 & 0.000 & - \\
\hline 10633.2 & $2 \mathrm{~h}$ & $\mathrm{~T}$ & 2.45 & 4.56 & 0.005 & - \\
\hline
\end{tabular}

a Mean $\mathrm{m} / \mathrm{z}$ value of each peak. ${ }^{\mathrm{b}}$ Duration of treatment. ${ }^{\mathrm{c}} \mathrm{N}$ and $\mathrm{T}$ denote normal and tumor, respectively. ${ }^{\mathrm{d}}$ Mean normalized intensity for untreated samples. ${ }^{\mathrm{e}}$ Mean normalized intensity for treated samples. ${ }^{\mathrm{f}} \mathrm{p}$-values were calculated by Mann-Whitney U statistics for each peak. ${ }^{\mathrm{g}} \mathrm{X}$ denotes that it was found significant by permutation and - denotes that it was not.

Table V. SELDI-TOF-MS analytical reproducibility.

\begin{tabular}{lcccc}
\hline Fraction & Pellet & Supernatant & Pellet & Supernatant \\
\hline Array & IMAC30 & IMAC30 & CM10 & CM10 \\
Replicates $^{\mathrm{a}}$ & 16 & 19 & 18 & 21 \\
Peaks $^{\mathrm{b}}$ & 21 & 18 & 12 & 13 \\
$\mathrm{CV}^{\mathrm{c}}$ & $16.6 \%$ & $24.0 \%$ & $29.0 \%$ & $17.4 \%$ \\
\hline
\end{tabular}

${ }^{a}$ Number of QC samples analyzed. ${ }^{b}$ Number of peak clusters detected using the Biomarker Wizard tool. ${ }^{\mathrm{c}}$ Mean coefficient of variance for all detected peaks.

arrays. Each fraction was analyzed on one ProteinChip array type at a time, resulting in a total of four individual runs. The achieved analytical reproducibility varied between 16.6 and $29 \%$, as measured by means of mean CV on a number of quality control samples analyzed in every run (Table V).

Excluded samples. The number of included samples in the various experiments was reduced following exclusion of detected outliers. From experiment 1, where the animals received treatment for 6 days, all three replicates of two tumor samples were excluded from all subsets; one treated and one untreated. From experiment 2, where the animals received treatment for 14 days, all comparisons of treated and untreated tumors were based on CM10 data alone, due to a large number of excluded tumor samples from the IMAC subsets. From the same experiment, 1 analytical replicate of an untreated tumor sample was excluded from the CM10 datasets.

From experiment 3, where the animals received a single dose vandetanib 19 days after implantation, all comparisons of treatment effects after $24 \mathrm{~h}$ were carried out only on data acquired on CM10 ProteinChip arrays, as a consequence of many exclusions from the IMAC datasets.

Overview of untreated samples. PCA on complete protein expression profiles $(\mathbf{X})$ from untreated samples revealed a large variation between the different types of samples included in the study (Fig. 2). Each individual sample group clustered fairly well together and there was a distinction between the tumor samples and non-tumor samples. A difference related to tumor progression could also be observed, where tumor samples from animals with tumors implanted for 12 days clustered closer the non-tumor samples than those from animals with tumors implanted for 20 days. Furthermore, a trend was detectable among the overlapping groups of nontumor samples, where samples from tumor-bearing rats clustered closer to the tumor samples as compared to the samples from sham operated, and tumor-free rats.

Effects of treatment. An overview of the mean normalized and binned spectra of tumor samples from all experiment groups, both from treated and untreated control animals, is presented in Fig. 3. The animals in the first experiment group, with tumors implanted for 12 days receiving daily doses of vandetanib for the last 6 days, displayed 13 peaks with a significantly altered expression (5 in normal and 8 in tumor tissue) between treated and untreated control animals (Tables I-IV). The cross validated scores from the final, 


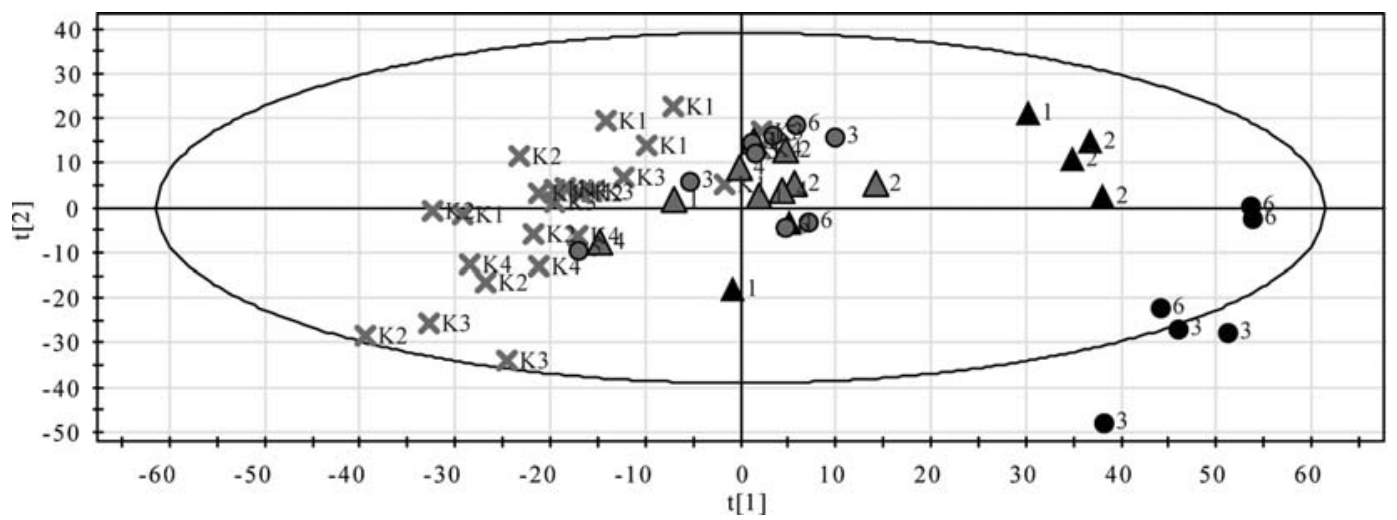

Figure 2. Overview of untreated samples. The x- and y-axes represent the first and second component, respectively, of a PCA model based on complete expression profiles, from the supernatant fraction analyzed on IMAC arrays, in the 1.8-10 kDa mass range. The colors and shapes denote different types of samples; gray diagonal crosses (x), samples from tumor-free, sham operated rats. The triangles and circles represent samples from experiment 1 (sacrificed 12 days after implantation) and 2 (sacrificed 20 days after implantation), respectively, where black symbols denote tumor samples and gray contralateral normal samples. Each sample is labeled with its corresponding animal number.

A

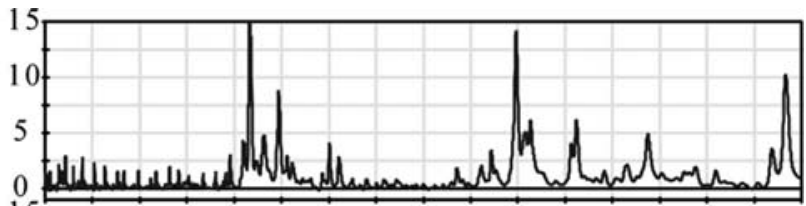

B

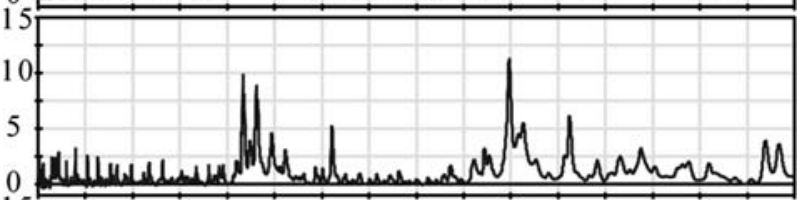

C

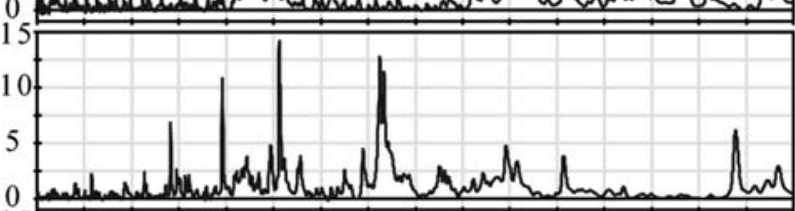

D$$
15
$$

15

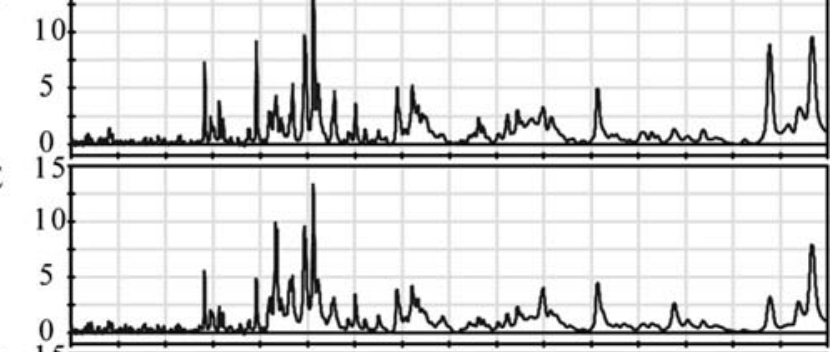

$\mathrm{F}$

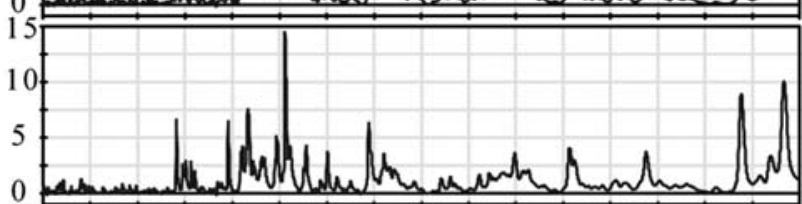

G

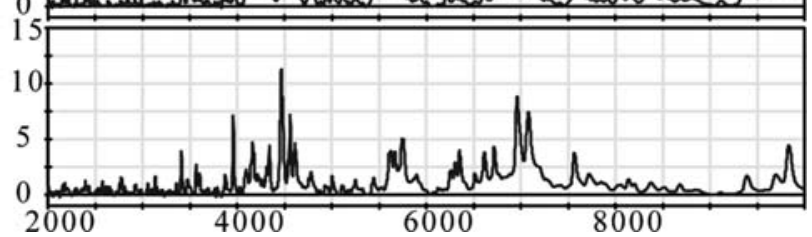

Figure 3. Mean protein expression profiles, based on normalized and binned data from the pellet fraction of tumor samples, analyzed on CM10 ProteinChip arrays. (A and B) Untreated and treated samples, respectively, from animals in experiment 1 (sacrificed 12 days after implantation). (C) Untreated animals sacrificed 20 days after implantation, used as control group for both experiments 2 and 3. (D-F) Spectra from treated animals in experiment group 3 , where the animals were treated with a single dose of vandetanib 19 days after implantation and then sacrificed after 2 (D), 8 (E) and 24 (F) h. (G) Results from the analysis of the treated animals in experiment 2 (sacrificed 20 days after implantation).
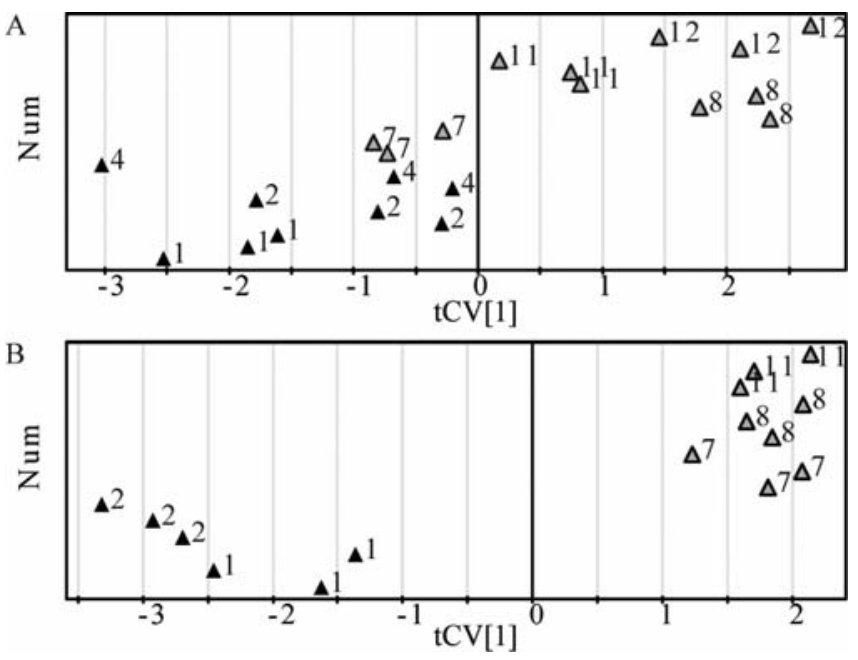

Figure 4. Discrimination between treated and untreated animals, based on peaks found to be significantly affected after 6 days of treatment. The X-axis represents the first cross validated component from the OPLS-DA model based on significantly altered peaks in normal (A) and tumor (B) tissue, using treatment as a single response variable. The y-axis represents unique numbers assigned to each sample. Each sample is labeled with its corresponding animal number. The colors denote treatment, where black represents untreated control and light gray represents treated animals.

UV-scaled, OPLS-DA models, based on the combined expression pattern of these peaks, displayed a clear separation of the tumor samples as a result of treatment. The equivalent separation was less evident among the normal tissue samples (Fig. 4).

Twenty days after tumor implantation, following 14 days of treatment, 39 peaks in total were detected with a significantly altered intensity between treated and untreated animals. Ten of these peaks were common between the tissue types, 22 were specific for normal tissue samples and 7 specific for tumor samples (Tables I-IV). The cross-validated scores from the final, UV-scaled, OPLS-DA models, based on the combined expression pattern of these peaks, displayed clear separations in both tissue types (Fig. 5).

Furthermore, we compared protein expression in normal and tumor tissue at 3 different time-points (2, 8 and $24 \mathrm{~h}$ ) after a single dose of vandetanib in animals with tumors 

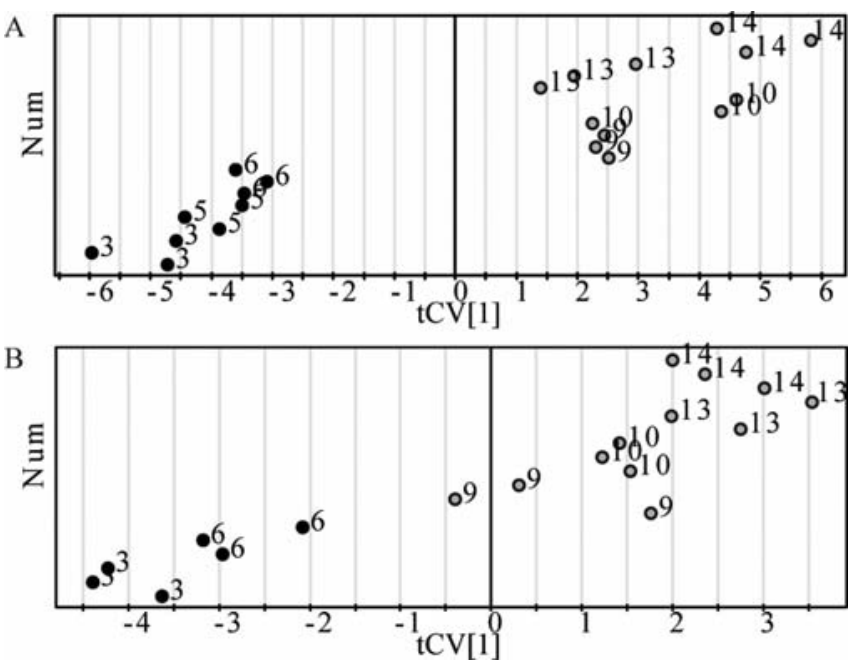

Figure 5. Discrimination between untreated control animals and animals treated for 14 days. The $\mathrm{x}$-axis represents the first cross validated component from the OPLS-DA model based on peaks found significantly altered in normal (A) and tumor (B) tissue. The y-axis represents unique numbers assigned to each sample. Each sample is labeled with its corresponding animal number. The colors denote treatment, where black represents untreated control and light gray represents treated animals.
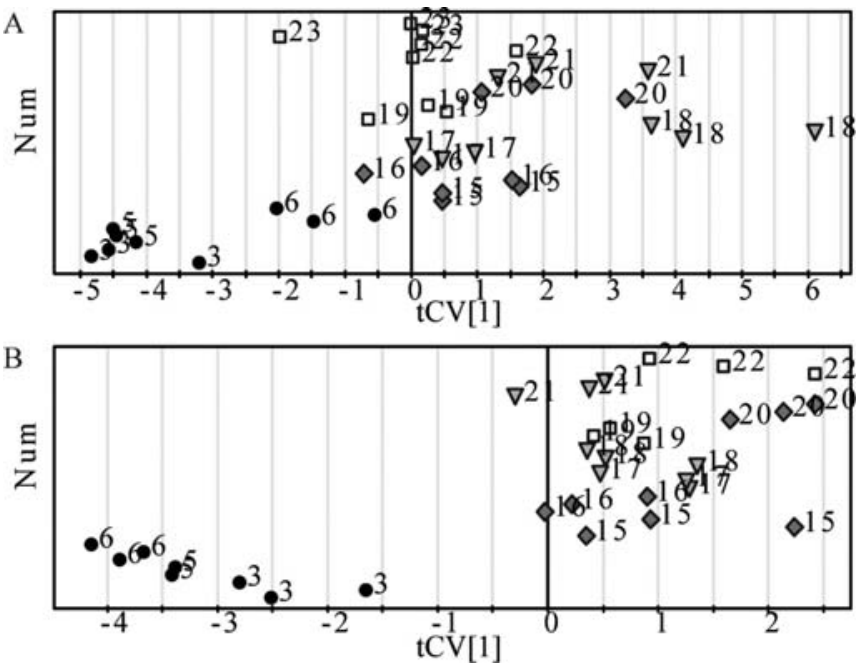

Figure 6. Effects of a single dose of vandetanib were examined at three different time-points post treatment, represented by different colors and shapes: black circles, untreated controls; dark gray diamonds. $2 \mathrm{~h}$; light gray inverted triangles, $8 \mathrm{~h}$ and unfilled boxes, $24 \mathrm{~h}$. The figure illustrates the discrimination between each group of treated animals and the untreated control animals, in normal (A) and tumor (B) tissue, by studying the expression pattern of all peaks found significantly changed in at least one of the treatment groups (as compared to untreated controls) by means of OPLS-DA, using treatment as a single response. The $\mathrm{x}$-axis represents the first cross validated component from the OPLS-DA model. The y-axis represents unique numbers assigned to each sample. Each sample is labeled with its corresponding animal number.

implanted for 19 days (experiment 3). From these three comparisons carried out in both tissue types, a total of 40 peaks were detected showing a significantly altered intensity, after at least one of the analyzed time-points, in treated tissue as compared to untreated tissue of the same sort (tumor or normal). Nineteen of these were found specifically in tumor tissue, 17 specifically in normal tissue and 4 were found in both tissue types (Tables I-IV). In the cross-validated scores from the final, UV-scaled, OPLS-DA models based on these peaks it was clear that each of the treated groups were different from the control group. The separation was more evident among the tumor samples than among the samples from normal tissue, where a slight overlap was seen (Fig. 6).

\section{Discussion}

In the present study, a distinct and time-dependent alteration in the protein expression pattern of malignant glioma and normal brain is reported after vandetanib treatment, as examined by a mass-spectrometric method and multivariate data analysis. A list of peaks significantly affected by treatment is presented and may represent future candidate biomarkers for treatment response. To our knowledge, this is the first study that investigates the influence of a tyrosine kinase inhibitor on the protein expression pattern in an in vivo glioma model.

Today, there are few biological markers in clinical use for brain tumor treatment decision-making. For glioblastoma treatment, methylation of the O6-methylguanin-DNA methyltransferase (MGMT) gene, predicts a significantly better response to treatment with alkylating agents $(28,29)$. In tumors of oligodendroglial origin, loss of heterozygosity ( $\mathrm{LOH}) 1 \mathrm{p}$ and $19 \mathrm{q}$ appears to harbor prognostic as well as predictive information $(30,31)$. These novel biomarkers are now being integrated in clinical trials and everyday clinical practice. However, in the search for new glioblastoma treatments, specific and reliable markers for early treatment response are urgently needed. This is accentuated when it comes to the development of anti-angiogenesis treatments, aiming to inhibit tumor vascularisation. Here, traditional indicators of response, such as radiological tumor size evaluations, are not per se indicators of successful inhibition of angiogenesis. At present, there are no reliable markers of response to anti-angiogenesis treatment, but a few candidates have been proposed. In the experimental situation, micro-vascular density (MVD) has been used to assess effects of anti-angiogenesis treatment, though this usage is disputed (32). Another suggested measure of anti-angiogenesis treatment effects is by assessment of the number of circulating endothelial cells (CEC) and circulating endothelial progenitor cells (CEP) $(33,34)$. The usefulness of these tools was further evaluated by Beaudry et al, who describe differential changes in the levels of CEC and CEP following treatment with vandetanib (35), the same tyrosine kinase inhibitor that was used in the present study. Moreover, MRI based radiological methods for imaging of the tumor vascular compartment have been reported to add predictive information for anti-angiogenesis treatment $(36,37)$. These MRI methods are promising and have the advantage of being non-invasive. Even though changes in CEC/CEP levels or MRI perfusion parameters may reflect successful angiogenesis inhibition per se they are less likely to be specific for a particular angio-genesis inhibitor.

Proteomic protocols are attractive methods to find new biomarkers. The biological and physiological effects of antiangiogenesis treatment are several, including inhibition of endothelial cell proliferation and functional normalization of the tumor vascular bed $(7,20,38)$. Biomarkers for anti- 
angiogenesis treatment may therefore be found in different cell-signaling pathways directly or indirectly affected by a specific treatment. A proteomic screening approach, employing a method such as SELDI-TOF-MS, then holds the potential to discover novel protein markers as well as expression patterns of interest. The capacity of this method to analyze samples from cancer patients has been established previously $(39,40)$. It has also been utilized to detect treatment-induced changes in the urinary proteome of patients with polycystic kidney diseases (41) as well as by our group for studying effects of radiotherapy in rat glioma (26). However, most previous studies on treatment-induced changes in glioma protein expression patterns have been performed in vitro $(22,23,42)$. in vivo, however, tumor cells are in continuous interaction with stromal cells (43), which will impact on the tumor cells' protein expression pattern. Therefore, in the search for biomarkers we believe that it is important to study treatment effects in vivo.

Application of multivariate analysis methods was essential to efficiently handle the large amounts of information generated. We also believe this approach opens for the possibility to find panels of markers displaying specific patterns of expression that may be indicative of a specific biological state, even when none of the markers individually would be. When working with supervised data analysis methods, such as OPLS-DA, there is an inherent risk of generating false-positive findings due to overestimation of the data. In this study we have minimized this risk by use of extensive validation, including cross-validation, response permutation and Mann-Whitney statistics. Essentially, the data analysis was divided into two separate parts; initial analysis of complete expression profiles, carried out individually for each data subset, followed by analysis of selected peaks from all subsets (Xb). For the second step, two complementary approaches were used; investigation of OPLS-DA loadings and permutation analysis. The two approaches complement each other in the sense that in cases when the OPLS-DA model based on the $\mathbf{X b}$-matrix failed to completely divide the treatment effects into the second two predictive components, there was a risk of missing potentially important variables by studying the p(corr)-loading vectors of these two components alone. At the same time, the permutation test is very stringent and using only this approach could lead to missing possibly important peaks.

In the present study we report a total of 78 peaks in our spectra whose intensities changed significantly following treatment with vandetanib. There is a risk that Tables I-IV, listing peaks significantly affected by treatment, may contain overlapping information, as a consequence of the possibility that the same molecule can be present in both sample fractions analyzed and that it can bind to both of the used array types. However, the peaks found to be affected by a single dose were different ones at different time-points after treatment, indicating a dynamic immediate response. Furthermore, we could detect distinct differences between protein profiles from untreated tumors at different time-points after implantation (Fig. 2). Given that these tumor groups display different protein expression characteristics to begin with, one would expect to find discrepancies between the groups also among the treatment induced changes, which was indeed the case
(Tables I-IV). The complexity and dynamics of the protein expression is also demonstrated visually in Fig. 3, which further stresses the importance of designing experiments including control groups for each individual time-point of interest. In many previous proteomic studies of glioma in vitro the analyses of protein expression have been done at a single time-point after treatment $(22,23,42,44)$. Based on our findings, together with the information from the earlier referred study (26), we believe that the issue of proteomic response in malignant glioma to treatment is complex and that potential markers may be expressed differently in relation to timing of treatment and sampling of specimens.

Our findings demonstrated distinctly different protein expression patterns in tumor tissue as the tumor progresses over time. This is in concordance with previous findings in the same animal model (26). In the present study, the analysis has been expanded as compared to the previously published study, to include separate, thorough investigations of different durations of treatment in both tumor and contralateral normal tissue. Taken together, SELDI-TOF-MS in combination with appropriate multivariate statistical methods seem well suited for screening studies of treatment effects following various treatments and hold the potential to discover novel markers.

Interestingly, we found significant differences in the protein expression profile induced by vandetanib treatment also in normal brain. As shown in Fig. 2, the normal tissue contralateral to the BT4C tumor seems to have a slightly different protein expression profile than normal brain tissue from tumor-free, sham-operated rats. The normal tissue from tumor-bearing rats clustered more towards the tumor samples. This may be explained by the presence of invading tumor cells or by a physiologic effect induced by the growing tumor in the contralateral hemisphere. The treatment effects observed in the normal brain of rats with BT4C tumors may be an indirect effect due to altered physiology after treatment or a direct effect on invading glioma cells. However, it cannot be excluded that the observed changes in the protein profile of normal brain, at least in part, may be a direct effect on normal brain tissue. Vandetanib has in clinical studies on pulmonary carcinoma and breast carcinoma not been reported to exert any obvious neurotoxic side-effects and only a few cases with mild headache were reported $(45,46)$. Notably, the animals in this and previous series have not shown any apparent neurologic side-effects $(13,21)$.

In conclusion, the present study reports a significantly altered protein expression pattern in malignant glioma and normal brain, following anti-angiogenesis treatment with the tyrosine kinase inhibitor vandetanib. A number of candidate biomarkers for treatment response were detected for further characterization. We believe that clinically relevant biomarkers for anti-angiogenesis treatment response in glioblastoma may be important in the future development of new treatment combinations.

\section{Acknowledgements}

We wish to express our gratitude to Håkan Hedman for proofreading the manuscript. Charlotte Nordström and Mikael Kimdal are acknowledged for skilful technical assistance and late Britt-Inger Gladzki for taking good care of the animals. 
We also like to thank AstraZeneca for providing vandetanib (ZD6474). This work was supported by grants from Lion's Cancer Research foundation, Umeå University, Sweden, The Research Foundation of Umeå University, Sweden and Stiftelsen Dagmar Ferbs minnesfond, Karolinska Institute, Stockholm, Sweden. Preliminary parts of this manuscript were presented at the 8th European Association of NeuroOncology congress, September 12-14, 2008. For the duration of this study, Prof. R. Henriksson was working as a consultant for AstraZeneca, the company developing vandetanib.

\section{References}

1. Stupp R, Dietrich PY, Ostermann Kraljevic S, et al: Promising survival for patients with newly diagnosed glioblastoma multiforme treated with concomitant radiation plus temozolomide followed by adjuvant temozolomide. J Clin Oncol 20: 1375-1382, 2002.

2. Hanahan D and Weinberg RA: The hallmarks of cancer. Cell 100: 57-70, 2000.

3. Leon SP, Folkerth RD and Black PM: Microvessel density is a prognostic indicator for patients with astroglial brain tumors. Cancer 77: 362-372, 1996.

4. Yao Y, Kubota T, Takeuchi H and Sato K: Prognostic significance of microvessel density determined by an anti-CD105/endoglin monoclonal antibody in astrocytic tumors: comparison with an anti-CD31 monoclonal antibody. Neuropathology 25: 201-206, 2005

5. Plate KH, Breier G, Millauer B, Ullrich A and Risau W: Upregulation of vascular endothelial growth factor and its cognate receptors in a rat glioma model of tumor angiogenesis. Cancer Res 53: 5822-5827, 1993.

6. Johansson M, Brannstrom T, Bergenheim AT and Henriksson R: Spatial expression of VEGF-A in human glioma. J Neurooncol 59: 1-6, 2002.

7. Jain RK, Di Tomaso E, Duda DG, Loeffler JS, Sorensen AG and Batchelor TT: Angiogenesis in brain tumours. Nat Rev Neurosci 8: 610-622, 2007.

8. Cheng SY, Huang HJ, Nagane M, et al: Suppression of glioblastoma angiogenicity and tumorigenicity by inhibition of endogenous expression of vascular endothelial growth factor. Proc Natl Acad Sci USA 93: 8502-8507, 1996.

9. Kim KJ, Li B, Winer J, et al: Inhibition of vascular endothelial growth factor-induced angiogenesis suppresses tumour growth in vivo. Nature 362: 841-844, 1993.

10. Kunkel P, Ulbricht U, Bohlen P, et al: Inhibition of glioma angiogenesis and growth in vivo by systemic treatment with a monoclonal antibody against vascular endothelial growth factor receptor-2. Cancer Res 61: 6624-6628, 2001.

11. Gomez-Manzano C, Holash J, Fueyo J, et al: VEGF trap induces antiglioma effect at different stages of disease. Neurooncology (In pres).

12. De Bouard S, Herlin P, Christensen JG, et al: Antiangiogenic and anti-invasive effects of sunitinib on experimental human glioblastoma. Neurooncology 9: 412-423, 2007.

13. Sandstrom M, Johansson M, Andersson U, Bergh A, Bergenheim AT and Henriksson R: The tyrosine kinase inhibitor ZD6474 inhibits tumour growth in an intracerebral rat glioma model. Br J Cancer 91: 1174-1180, 2004.

14. Rich JN, Sathornsumetee S, Keir ST, et al: ZD6474, a novel tyrosine kinase inhibitor of vascular endothelial growth factor receptor and epidermal growth factor receptor, inhibits tumor growth of multiple nervous system tumors. Clin Cancer Res 11: 8145-8157, 2005.

15. Goldbrunner RH, Bendszus M, Wood J, Kiderlen M, Sasaki M and Tonn JC: PTK787/ZK222584, an inhibitor of vascular endothelial growth factor receptor tyrosine kinases, decreases glioma growth and vascularization. Neurosurgery 55: 426-432, 2004.

16. Vredenburgh JJ, Desjardins A, Herndon JEII, et al: Phase II trial of bevacizumab and irinotecan in recurrent malignant glioma. Clin Cancer Res 13: 1253-1259, 2007.

17. Henriksson R, Bergstrom P, Johansson M and Sandstrom M: Enigma of a rapid introduction of antiangiogenic therapy with bevacizumab in glioblastoma: a new era in the treatment of malignant brain tumours? Acta Oncol 48: 6-8, 2009.
18. Poulsen HS, Grunnet K, Sorensen M, et al: Bevacizumab plus irinotecan in the treatment patients with progressive recurrent malignant brain tumours. Acta Oncol 48: 52-58, 2009.

19. Hennequin LF, Stokes ES, Thomas AP, et al: Novel 4-anilinoquinazolines with C-7 basic side chains: design and structure activity relationship of a series of potent, orally active, VEGF receptor tyrosine kinase inhibitors. J Med Chem 45: 1300-1312, 2002.

20. Wedge SR, Ogilvie DJ, Dukes M, et al: ZD6474 inhibits vascular endothelial growth factor signaling, angiogenesis, and tumor growth following oral administration. Cancer Res 62: 4645-4655, 2002.

21. Sandstrom M, Johansson M, Bergstrom P, Bergenheim AT and Henriksson R: Effects of the VEGFR inhibitor ZD6474 in combination with radiotherapy and temozolomide in an orthotopic glioma model. J Neurooncol 88: 1-9, 2008.

22. Puchades M, Nilsson CL, Emmett MR, et al: Proteomic investigation of glioblastoma cell lines treated with wild-type p53 and cytotoxic chemotherapy demonstrates an association between galectin-1 and p53 expression. J Proteome Res 6: 869-875, 2007.

23. Trog D, Fountoulakis M, Friedlein A and Golubnitschaja O: Is current therapy of malignant gliomas beneficial for patients? Proteomics evidence of shifts in glioma cells expression patterns under clinically relevant treatment conditions. Proteomics 6: 2924-2930, 2006

24. Bergenheim AT, Elfverson J, Gunnarsson P-O, Edman K, Hartman M and Henriksson R: Cytotoxic effect and uptake of estramustine in a rat glioma model. Int J Oncol 5: 293-299, 1994.

25. Johansson M, Henriksson R, Bergenheim AT and Koskinen LO: Interleukin-2 and histamine in combination inhibit tumour growth and angiogenesis in malignant glioma. Br J Cancer 83: 826-832, 2000.

26. Wibom C, Pettersson F, Sjostrom M, Henriksson R, Johansson M and Bergenheim AT: Protein expression in experimental malignant glioma varies over time and is altered by radiotherapy treatment. Br J Cancer 94: 1853-1863, 2006.

27. Bylesjö M, Rantalainen M, Cloarec O, Nicholson JK, Holmes E and Trygg J: OPLS discriminant analysis: combining the strengths of PLS-DA and SIMCA classification. J Chemometrics 20: 341-351, 2006.

28. Hegi ME, Diserens AC, Gorlia T, et al: MGMT gene silencing and benefit from temozolomide in glioblastoma. N Engl J Med 352: 997-1003, 2005.

29. Hegi ME, Liu L, Herman JG, et al: Correlation of O6-methylguanine methyltransferase (MGMT) promoter methylation with clinical outcomes in glioblastoma and clinical strategies to modulate MGMT activity. J Clin Oncol 26: 4189-4199, 2008.

30. Cairncross JG: Imaging molecular signatures in oligodendroglioma. Clin Cancer Res 10: 7109-7111, 2004

31. Jeon YK, Park K, Park CK, Paek SH, Jung HW and Park SH: Chromosome $1 \mathrm{p}$ and $19 \mathrm{q}$ status and p53 and p16 expression patterns as prognostic indicators of oligodendroglial tumors: a clinicopathological study using fluorescence in situ hybridization. Neuropathology 27: 10-20, 2007.

32. Hlatky L, Hahnfeldt P and Folkman J: Clinical application of antiangiogenic therapy: microvessel density, what it does and doesn't tell us. J Natl Cancer Inst 94: 883-893, 2002.

33. Willett CG, Boucher Y, Di Tomaso E, et al: Direct evidence that the VEGF-specific antibody bevacizumab has antivascular effects in human rectal cancer. Nat Med 10: 145-147, 2004.

34. Willett CG, Boucher Y, Duda DG, et al: Surrogate markers for antiangiogenic therapy and dose-limiting toxicities for bevacizumab with radiation and chemotherapy: continued experience of a phase I trial in rectal cancer patients. J Clin Oncol 23: 8136-8139, 2005.

35. Beaudry P, Force J, Naumov GN, et al: Differential effects of vascular endothelial growth factor receptor-2 inhibitor ZD6474 on circulating endothelial progenitors and mature circulating endothelial cells: implications for use as a surrogate marker of antiangiogenic activity. Clin Cancer Res 11: 3514-3522, 2005.

36. Akella NS, Twieg DB, Mikkelsen T, et al: Assessment of brain tumor angiogenesis inhibitors using perfusion magnetic resonance imaging: quality and analysis results of a phase I trial. J Magn Reson Imaging 20: 913-922, 2004.

37. Batchelor TT, Sorensen AG, Di Tomaso E, et al: AZD2171, a pan-VEGF receptor tyrosine kinase inhibitor, normalizes tumor vasculature and alleviates edema in glioblastoma patients. Cancer Cell 11: 83-95, 2007. 
38. Tong RT, Boucher Y, Kozin SV, Winkler F, Hicklin DJ and Jain RK: Vascular normalization by vascular endothelial growth factor receptor 2 blockade induces a pressure gradient across the vasculature and improves drug penetration in tumors. Cancer Res 64: 3731-3736, 2004.

39. Ball G, Mian S, Holding F, et al: An integrated approach utilizing artificial neural networks and SELDI mass spectrometry for the classification of human tumours and rapid identification of potential biomarkers. Bioinformatics 18: 395-404, 2002.

40. Koopmann J, Zhang Z, White N, et al: Serum diagnosis of pancreatic adenocarcinoma using surface-enhanced laser desorption and ionization mass spectrometry. Clin Cancer Res 10: 860-868, 2004.

41. Moreno S, Ibraghimov-Beskrovnaya O and Bukanov NO Serum and urinary biomarker signatures for rapid preclinical in vivo assessment of cdk inhibition as a therapeutic approach for PKD. Cell Cycle 7: 1856-1864, 2008.

42. Demasi MA, Montor WR, Ferreira GB, Pimenta DC, Labriola L and Sogayar MC: Differential proteomic analysis of the antiproliferative effect of glucocorticoid hormones in ST1 rat glioma cells. J Steroid Biochem Mol Biol 103: 137-148, 2007.
43. Hoelzinger DB, Demuth T and Berens ME: Autocrine factors that sustain glioma invasion and paracrine biology in the brain microenvironment. J Natl Cancer Inst 99: 1583-1593, 2007.

44. Billecke C, Malik I, Movsisyan A, et al: Analysis of glioma cell platinum response by metacomparison of two-dimensional chromatographic proteome profiles. Mol Cell Proteomics 5: 35-42, 2006.

45. Heymach JV, Paz-Ares L, De Braud F, et al: Randomized phase II study of vandetanib alone or with paclitaxel and carboplatin as first-line treatment for advanced non-small-cell lung cancer. J Clin Oncol 26: 5407-5415, 2008.

46. Miller KD, Trigo JM, Wheeler C, et al: A multicenter phase II trial of ZD6474, a vascular endothelial growth factor receptor-2 and epidermal growth factor receptor tyrosine kinase inhibitor, in patients with previously treated metastatic breast cancer. Clin Cancer Res 11: 3369-3376, 2005. 